\title{
REGULARITY OF GROUP VALUED BAIRE AND BOREL MEASURES
}

\author{
K. SUNDARESAN ${ }^{1}$ AND PETER W. DAY 2
}

\begin{abstract}
It is known that a real valued measure (1) on the $\sigma$-ring of Baire sets of a locally compact Hausdorff space, or (2) on the Borel sets of a complete separable metric space is regular. Recently Dinculeanu and Kluvánek used regularity of nonnegative Baire measures to prove that any Baire measure with values in a locally convex Hausdorff topological vector space (TVS) is regular. Subsequently a direct proof of the same result was offered by Dinculeanu and Lewis. Here we show just as directly that any measure defined as in (1) or (2) is regular, even when it takes values in a Hausdorff topological group. In particular, when the group is a Hausdorff TVS, our result improves the Dinculeanu-KluvánekLewis theorem.
\end{abstract}

1. Introduction. Of the several proofs of regularity of Baire measures in the literature, the simplest seems to be the one offered in a recent paper of Dinculeanu and Lewis [2]. By bringing in the $p$-quasi variation, however, these authors make heavy use of the fact that the measures they consider take values in a Hausdorff locally convex space. We will show that in fact their result holds even when the range space is only a Hausdorff topological group. With minimal additional work we will also show that a group valued Borel measure on a complete separable metric space is also regular.

We briefly recall some notations and basic definitions required in the subsequent discussion.

In what follows, $G$ is always a Hausdorff topological group written multiplicatively, with identity element 1 , and $\mathfrak{F}^{\prime}$ denotes the filter of all neighborhoods of 1 . Let $\mathscr{R}$ be a ring of sets. A function $m: \mathscr{R} \rightarrow G$ is said to be a measure if $m\left(\bigcup_{i=1}^{\infty} A_{i}\right)=\lim _{n \rightarrow \infty} m\left(A_{1}\right) \cdots m\left(A_{n}\right)$ exists in $G$ whenever $\left\{A_{i}\right\}_{i=1}$ is a sequence of pairwise disjoint sets in $\mathscr{R}$ such that

Received by the editors November 5, 1971 and, in revised form, March 13, 1972. AMS 1969 subject classifications. Primary 2210, 2850; Secondary 2635, 2810.

Key words and phrases. Regular measure, group valued measure, Baire measure, Borel measure.

${ }^{1}$ The research work of the author was supported in part by the Scaife Faculty Grant 1971 administered by Carnegie-Mellon University.

2 Research in part supported by NSF Grant GU-2056.

(c) American Mathematical Society 1973 
$\bigcup_{i \geqq 1} A_{i} \in \mathscr{R}$. It is verified that (1) when $m$ is a measure and $\left\{A_{i}\right\}_{i \geqq 1}$ is as above, then $\lim _{n \rightarrow \infty} m\left(A_{n}\right)=1$; and (2) if $m: \mathscr{R} \rightarrow G$ is finitely multiplicative then $m$ is a measure iff $\lim _{i \rightarrow \infty} m\left(B_{i}\right)=1$ whenever $B_{i} \downarrow \varnothing, B_{i} \in \mathscr{R}$. Further in passing we remark that the subgroup generated by the range of a group valued measure is abelian. Since we do not require this fact here, the verification of this fact is left to the reader. For a discussion of some problems concerning group valued measures, we refer to Sion [5].

Let $X$ be a Hausdorff topological space, and let $\mathscr{R}$ be a ring of subsets of $X$. A measure $m: \mathscr{R} \rightarrow G$ is called regular at $A \in \mathscr{R}$ if for every $U \in \mathscr{N}$ there is a compact set $K \in \mathscr{R}$ and an open set $G \in \mathscr{R}$ such that $K \subset A \subset G$, and $m(S) \in U$ whenever $S \subset G \backslash K, S \in \mathscr{R}$. The pair $(K, G)$ is then said to work for $(A, U)$. The measure $m$ is called regular on $\mathscr{R}$ if it is regular at each member of $\mathscr{R}$.

2. The lemmas. Before proceeding to the main theorem we state two useful results. The first one is due to Gould [4] for Banach space valued measures. The second one is a principle which seems to be known. We sketch a proof here for the sake of completeness. Recall that a ring is called a $\delta$-ring if it is closed for countable intersections.

Lemma 1. Let $\mathscr{R}$ be a $\delta$-ring and let $m: \mathscr{R} \rightarrow G$ be a measure. If $A_{n} \downarrow \varnothing$, $A_{n} \in \mathscr{R}$, then for each $U \in \mathscr{N}$ there is a natural number $N$ such that if $S \subset A_{N^{\prime}}$ and $S \in \mathscr{R}$, then $m(S) \in U$.

Proof. Suppose that the lemma is false. Then there is a sequence $A_{n} \downarrow \varnothing, A_{n} \in \mathscr{R}$, a $U \in \mathscr{N}$, and a sequence $\left\{S_{n}\right\}_{n>1}$ in $\mathscr{R}$ such that $S_{n} \subset A_{n}$ and $m\left(S_{n}\right) \notin U$ for all $n \geqq 1$. Let $V, W \in \mathcal{N}^{\prime \prime}$ be such that $V \cdot W \subset U$, and let $n_{1}=1$. If $n_{k}$ is defined, then, since $m\left(S_{n_{k}} \cap A_{i}\right) \rightarrow 1$ as $i \rightarrow \infty$, there is an $n_{k+1}>n_{k}$ such that $m\left(S_{n_{k}} \cap A_{n_{k+1}}\right) \in W$. Let

$$
B_{k}=S_{n_{k}} \backslash\left(S_{n_{k}} \cap A_{n_{k+1}}\right) \text {. }
$$

Then $B_{k}=S_{n_{k}} \cap\left(A_{n_{k}} \backslash A_{n_{k+1}}\right) \subset A_{1}$ and $B_{k} \cup\left(S_{n_{k}} \cap A_{n_{k+1}}\right)=S_{n_{k}}$. Hence

$$
m\left(B_{k}\right) \cdot m\left(S_{n_{k}} \cap A_{n_{k+1}}\right)=m\left(S_{n_{k}}\right) \notin U,
$$

so $m\left(B_{k}\right) \notin V$. Now $\bigcup_{k \gg 1} B_{k} \in \mathscr{R}$ because $\bigcup_{k \gg 1} B_{k} \subset A_{1}$ and $\mathscr{R}$ is a $\delta$-ring. But the sets $\left\{B_{k}\right\}_{k \supseteq 1}$ are disjoint, so $m\left(B_{k}\right) \rightarrow 1$ as $k \rightarrow \infty$. This contradicts $m\left(B_{k}\right) \notin V$ for all $k \geqq 1$ and completes the proof of the lemma.

LEMMA 2. If $\mathscr{R}$ is a $\sigma$-ring [ $\delta$-ring] of sets generated by a class $\mathscr{D}$, and if $m: \mathscr{R} \rightarrow G$ is a measure which is regular at each member of $\mathscr{D}$, then $m$ is regular on $\mathscr{R}$.

Proof. Let $\mathscr{A}=\{\mathscr{A} \in \mathscr{R}: m$ is regular at $A\}$. We show first that $\mathscr{A}$ is a ring. Let $A_{1}, A_{2} \in \mathscr{A}$, let $U \in \mathscr{N}$, and let $V_{1}, V_{2} \in \mathscr{N}$ be such that 
$V_{1} \cdot V_{2} \subset U$. Let $\left(K_{i}, G_{i}\right)$ work for $\left(A_{i}, V_{i}\right), i=1,2$. Then as in [2, p. 93, (b)] it is verified that $\left(K_{1} \cup K_{2}, G_{1} \cup G_{22}\right)$ works for $\left(A_{1} \cup A_{2}, U\right)$, and $\left(K_{1} \mid G_{2}\right.$, $\left.G_{1} \backslash K_{2}\right)$ works for $\left(A_{1} \backslash A_{2}, U\right)$. Thus $\mathscr{A}$ is a ring.

Next let $\mathscr{R}$ be a $\delta$-ring. We show that $\mathscr{A}$ is a $\delta$-ring. Suppose $A_{n} \downarrow A$, $A_{n} \in \mathscr{A}$, and $U \in \mathscr{V}^{\circ}$. Let $V_{0} \in \mathscr{A}^{\circ}$ be such that $V_{0} \cdot V_{0} \subset U$. By induction there is a sequence $\left\{V_{i}\right\}_{i \geq 1} \subset \mathcal{N}$ such that $V_{i+1} \cdot V_{i+1} \subset V_{i}, i \geqq 0$. Then $V_{1} \cdots V_{n} \subset V_{0}$ for all $n \geqq 1$. Let $\left(C_{i}, \mathcal{O}_{i}\right)$ work for $\left(A_{i}, V_{i}\right)$, let $K_{n}=$ $\bigcap_{i=1}^{n} C_{i}, G_{n}=\bigcap_{i=1}^{n} \mathcal{O}_{i}$, and $K=\bigcap_{n}{ }_{1} K_{n}$. By Lemma 1 there is an $N$ such that if $S \subset K_{Y} \backslash K$ and $S \in \mathscr{R}$ then $m(S) \in V_{0}$. It is then verified that $\left(K_{N}, G_{N}\right)$ works for $\left(A_{.}, V_{0}\right)$ so $\left(K, G_{.}\right)$works for $(A, U)$ as in [2, p. 93, (c)]. Thus $\mathscr{A}$ is a $\delta$-ring, so $\mathscr{A}=\mathscr{R}$.

If $\mathscr{R}$ is a $\sigma$-ring, then a similar argument shows that $A_{n} \uparrow A, A_{n} \in \mathscr{A}$ implies $A \in \mathscr{A}$, so $\mathscr{A}$ is a $\sigma$-ring, and again $\mathscr{A}=\mathscr{R}$.

3. The theorem. We are now ready to prove our theorem.

THEOREM. Let $X$ be a locally compact Hausdorff space [complete separable metric space], and let is be the $\sigma$-ring of Baire [Borel] sets in $X$. Then every $G$-valued measure on $\mathscr{B}$ is regular.

Proof. (1) Suppose $X$ is a locally compact Hausdorff space, and let $\mathscr{D}$ be the collection of all compact $G_{\delta}$ subsets of $X$. Let $K \in \mathscr{Z}$. It follows from [3, p. 296, Proposition 14] that there is a sequence $\left\{G_{n}\right\}_{n \geq 1}$ of open Baire sets such that $G_{n} \downarrow K$. Let $U \in \mathcal{A}$. By Lemma 1 there is a natural number $N$ such that $S \subset G_{.} \mid K, S \in \mathscr{B}$, implies $m(S) \in U$, i.e., $\left(K, G_{N}\right)$ works for $(K, U)$. Since $\mathscr{B}$ is the $\sigma$-ring generated by $\mathscr{Z}$, it follows from Lemma 2 that $m$ is regular.

(2) Now let $(X, d)$ be a complete, separable metric space, let $\mathscr{H}$ be the set of all closed subsets of $X$, let $A \in \mathscr{Z}$, let $U \in \mathcal{H}$, and let $V \in \mathscr{N}$ be such that $V \cdot V \subset U$. Since $A$ is closed, it is a $G_{j}$, so as in (1) there is an open set $G \supset A$ such that if $S \subset G \backslash A$ and $S \in \mathscr{B}$ then $m(S) \in V$.

We now construct a suitable compact set. Let $\left\{a_{i}\right\}_{i \cdots 1}$ be a countable, dense subset of $X$. For each natural number $n \geqq 1$, let

$$
B_{1 / n}\left(a_{i}\right)=\left\{y \in A: d\left(y, a_{i}\right) \leqq 1 / n\right\}
$$

and let $F_{k}(n)=\bigcup_{i=1}^{k} B_{1 / n}\left(a_{i}\right)$. From the denseness of $\left\{a_{i}\right\}_{i \geqq 1}$ it follows that for each $n, F_{k}(n) \uparrow_{k} A$. Let $W_{0} \in \mathscr{N}$ be such that $W_{0} \cdot W_{0} \subset V$, and define $\left\{W_{i}\right\}_{i \geqslant 1} \subset \mathcal{N}$ by induction so that $W_{i+1} \cdot W_{i+1} \subset W_{i}$ for all $i \geqq 0$. Then $W_{1} \cdots W_{n} \subset W_{0}$ for all $n \geqq 1$. By Lemma 1 there is a $k_{1}$ such that, if $S \subset A \backslash F_{k_{1}}(1)$ and $S \in \mathscr{B}$, then $m(S) \in W_{1}$. By induction there is a sequence $\left\{k_{i}\right\}_{i \gg 1}$ such that if $S \subset \bigcap_{k=1}^{n} F_{k_{i}}(i) \backslash \bigcap_{i=1}^{n+1} F_{k_{i}}(i)$ and $S \in \mathscr{B}$ then $m(S) \in$ $W_{n+1}$. Indeed, if $k_{1}, \cdots, k_{n}$ are defined, then

$$
\bigcap_{i=1}^{n} F_{k_{i}}(i) \backslash\left(\bigcap_{i=1}^{n} F_{k_{i}}(i) \cap F_{k}(n+1)\right) \downarrow_{k} \varnothing
$$


so the existence of $k_{n+1}$ follows from Lemma 1. Let $K_{n}=\bigcap_{i=1}^{n} F_{k_{i}}(i)$ and $K=\bigcap_{n \geqq 1} K_{n}$. It is verified that each sequence in $K$ has a convergent subsequence. Since $K$ is closed, $K$ is in fact compact. Since $K_{n} \downarrow K$ there is an $N$ such that if $S \subset K_{N} \backslash K$ and $S \in \mathscr{B}$ then $m(S) \in W_{0}$. Thus if $S \subset A \backslash K$ and $S \in \mathscr{B}$, then $S=S_{1} \cup \cdots \cup S_{N+1}$ where $S_{i} \in \mathscr{B}$ for $1 \leqq i \leqq N+1$, $S_{1} \subset A \backslash K_{1}, \quad S_{i} \subset K_{i-1} \backslash K_{i}$ for $2 \leqq i \leqq N$, and $S_{N+1} \subset K_{N} \backslash K$, so $m(S)=$ $m\left(S_{1}\right) \cdots m\left(S_{N+1}\right) \in W_{1} \cdots W_{N} W_{0} \subset V$. It follows that $(K, G)$ works for $(A, U)$. Thus $m$ is regular at every closed set, so by Lemma $2, m$ is regular at every Borel set, and the theorem is proved.

\section{REFERENCES}

1. N. Dinculeanu and I. Kluvánek, On vector measures, Proc. London Math. Soc. (3) 17 (1967), 505-512. MR 35 \#5571.

2. N. Dinculeanu and P. W. Lewis, Regularity of Baire measures, Proc. Amer. Math. Soc. 26 (1970), 92-94. MR 41 \#5588.

3. N. Dinculeanu, Vector measures, Internat. Series of Monographs in Pure and Appl. Math., vol. 95, Pergamon Press, Oxford; VEB Deutscher Verlag der Wissenschaften, Berlin, 1967. MR 34 \#6011b.

4. G. G. Gould, Integration over vector-valued measures, Proc. London Math. Soc. (3) 15 (1965), 193-225. MR 30 \#4894.

5. M. Sion, Outer measures with values in a topological group, Proc. London Math. Soc. (3) 19 (1969), 89-106. MR 39 \#398.

Department of Mathematics, Carnegie-Mellon University, Pittsburgh, Pennsylvania 15213 